\title{
HADRON STRUCTURE ON THE BACK OF AN ENVELOPE
}

\author{
A. W. THOMAS, R. D. YOUNG \\ Thomas Jefferson National Accelerator Facility \\ 12000 Jefferson Ave., \\ Newport News, VA 23185, USA \\ E-mail: awthomas@jlab.org \\ D. B. LEINWEBER \\ Special Research Center for the Subatomic Structure of Matter, and \\ Department of Physics, University of Adelaide, \\ Adelaide, SA 5005 Australia \\ E-mail: dleinweb@physics.adelaide.edu.au
}

\begin{abstract}
In order to remove a little of the mysticism surrounding the issue of strangeness in the nucleon, we present simple, physically transparent estimates of both the strange magnetic moment and charge radius of the proton. Although simple, the estimates are in quite good agreement with sophisticated calculations using the latest input from lattice QCD. We further explore the possible size of systematic uncertainties associated with charge symmetry violation (CSV) in the recent precise determination of the strange magnetic moment of the proton. We find that CSV acts to increase the error estimate by $0.003 \mu_{N}$ such that $G_{M}^{s}=-0.046 \pm 0.022 \mu_{N}$.
\end{abstract}

\section{Introduction}

The tremendous amount of experience that has been gained over the last 6 years, by studying the chiral extrapolation of lattice QCD data as a function of quark (or pion) mass, has led to very important insights into hadron structure. The two major lessons learned are that:

- The contribution of pion loops to hadron properties decreases very fast as the pion mass increases, becoming small and slowly varying for pion masses above about $500 \mathrm{MeV}^{1,2,3}$. As a corollary, it follows that the effect of kaon loops is always relatively small ${ }^{4}$ - an issue we shall return to soon in the context of strangeness form factors.

- Once the pion mass is of the order of $500 \mathrm{MeV}$ or higher, all hadron properties are smooth, slowly varying and essentially behave like 
the constituent quark model. The corollary to this is that if one wishes to build a constituent quark model of hadron structure, this is the mass region where it has a chance to work ${ }^{5}$ - far from the region of rapidly varying non-analytic behaviour associated with pions near the chiral limit.

The second lesson is of particular relevance to the understanding of duality, because in this mass region $\left(m_{\pi}>500 \mathrm{MeV}\right)$ the reconstruction of the valence parton distribution functions (PDFs) shows that each valence quark does indeed have a most likely momentum fraction around $1 / 3{ }^{6}$, precisely as one would expect in a naive constituent quark picture.

One of the remarkable things that became obvious from the beginning of these studies is the fact that the relatively naive cloudy bag model ${ }^{8,9}(\mathrm{CBM})$ did an astonishingly good job of describing the mass dependence of nucleon properties, whether it be the mass ${ }^{7}$, magnetic moments ${ }^{3}$ or moments of the $\mathrm{PDFs}^{6}$. This does not mean that the CBM is ideal, we see from the comparison against $G_{E n}{ }^{10,11}$, in particular, that the sharp surface of the MIT bag, upon which the CBM was built, is not such a good description of the valence quark structure, especially in the surface region ${ }^{12}$. However, what does seem to really describe the way hadron structure works is that there is a perturbative pion cloud around a core of confined valence quarks, confined in a region whose vacuum structure (the bag itself) differs from that of the QCD ground state.

The recent discovery that the chiral quark soliton model also yields the correct dependence of $m_{N}$ on $m_{\pi}{ }^{13}$ (apart from the incorrect chiral coefficient associated with the hedgehog approximation) is consistent with this interpretation, since even though one has to work extremely hard to construct the change in the vacuum structure inside the nucleon at the microscopic level, in the end it looks like a system of bound valence quarks surrounded by a perturbative pion cloud. The consequences of the change in vacuum structure inside the hadron, in terms of a contribution to $\bar{d} \neq \bar{u}$ and $\Delta \bar{u} \neq \Delta \bar{d}$ are in fact similar in both models ${ }^{14,15}$.

Further support for this idea comes from a remarkable discovery concerning the lattice QCD data for the nucleon and the $\Delta$ in both quenched (QQCD) and full QCD (QCD). In fact, one can describe the data with a simple fitting function, $\alpha+\beta m_{\pi}^{2}$ plus the pion self-energy loops which give rise to the leading (LNA) and next-to-leading non-analytic (NLNA) behaviour, evaluated using a finite range regulator of dipole form (with common mass parameter $\Lambda=0.8 \mathrm{GeV}$ ). The important discovery is that 
$\alpha$ and $\beta$ (for a given baryon) are the same within the fitting errors (a few percent) in QQCD and $\mathrm{QCD}^{2}$. This is the case even though for the $\Delta$ the self-energies differ by a factor of two, with the $\mathrm{N}-\Delta$ splitting being of order $500 \mathrm{MeV}$ in QQCD and only $300 \mathrm{MeV}$ in full QCD. It seems that the "core" or valence structure of these key baryons, defined by the particular value of $\Lambda=0.8 \mathrm{GeV}$, is essentially the same in QQCD and QCD. The implications of this for modeling hadron structure are yet to be fully investigated but once again a simple, perturbative treatment of the pion cloud contributions works exceptionally well.

One of the most impressive recent achievements of the chiral extrapolation program has been the determination of an extremely precise value for the strangeness magnetic moment, $G_{M}^{s}{ }^{16}$. This calculation used a combination of experimental data for the octet magnetic moments, the constraints of charge symmetry and chiral extrapolation of state of the art lattice data to obtain the ratios of the magnetic moments of either a valence $u$ quark in the proton and $\Sigma^{+}$or the valence $u$ quark in the neutron and the $\Xi^{0}$. By reducing the demands on lattice QCD to mere ratios, it is possible to dramatically reduce the systematic errors. The result, namely $G_{M}^{s}=-0.046 \pm 0.019 \mu_{N}$ is an order of magnitude more precise than any current experiment ${ }^{17,18,19,20,21}$ - a unique example in modern hadron physics. A similar analysis for the strangeness electric form factor, $G_{E}^{s}$, has not yet been possible, essentially because the experimental knowledge of octet baryon charge radii is nowhere near as precise as the knowledge of magnetic moments. However, our main purpose, to which we turn in the next section, is to use what we have learnt so far about hadron structure to make a "back of the envelope" estimate of both the strangeness electric and magnetic form factors. Then in the following section, we provide an estimate of the systematic uncertainty associated with charge symmetry violation in the recent precise determination of the strangeness magnetic moment of the nucleon ${ }^{16}$.

\section{Simple Model of the Strangeness Form Factors of the Proton}

We note first that there is no known example where the current quark masses show up in hadron physics undressed by non-perturbative glue. Thus the cost to make an $s-\bar{s}$ pair in the proton is of order 1.0 to $1.1 \mathrm{GeV}$ (twice the strange constituent quark mass). On the other hand, creating the $\bar{s}$ in a kaon and the $s$ in a $\Lambda$ costs only $0.65 \mathrm{GeV}$. (Note that the $\mathrm{N}$ to 
$K \Sigma$ coupling is considerably smaller than that for $\mathrm{N}$ to $K \Lambda$ and hence in this simple discussion we ignore it.) On these grounds alone we expect the virtual transition $\mathrm{N}$ to $K \Lambda$ to dominate the production of strangeness in the proton.

Next we estimate the probability for finding the $K \Lambda$ configuration. This probability is inversely proportional to the excitation energy squared. We work by comparison with the $\mathrm{N} \pi$ component of the nucleon wave function, for which there is a vast body of evidence that it is about $20 \%{ }^{22}$. Naively the transition $\mathrm{N}$ to $\mathrm{N} \pi$ costs $140 \mathrm{MeV}$, but with additional kinetic energy this is around $600 \mathrm{MeV}$ in total. Including similar kinetic energy for the $K \Lambda$ component as well, it costs roughly twice as much as $\mathrm{N} \pi$. Thus the $K \Lambda$ probability is of order $5 \%$.

\subsection{Strangeness radius}

We consider first the strangeness radius of the proton based on this $5 \% K \Lambda$ probability. In the CBM the radius of a $\Lambda$ bag is about $1 \mathrm{fm}$, which yields a mean square radius for the strange quark around $0.5 \mathrm{fm}^{2}$. As an estimate of the range of variation possible, we also take the bag radius $R=0.8 \mathrm{fm}$ with a corresponding mean square radius close to $0.36 \mathrm{fm}^{2}$. In order to estimate the contribution from the kaon cloud, we need to realize that in almost any chiral quark model the peak in the Goldstone boson wave function is at the confinement (bag) radius ${ }^{12,24}$. As long ago as 1980 this generated enormous interest in the precise measurement of $G_{E}^{n}{ }^{23}$. The meson field then decreases with a range between one over the energy cost of the Fock state and $1 /\left(m_{K}+m_{\Lambda}-m_{N}\right)$. Thus for $R=0.8 \mathrm{fm}$ we get a mean square radius for the $\bar{s}$ distribution of order $1 \mathrm{fm}^{2}$, while for $R=1 \mathrm{fm}$ we get about $1.4 \mathrm{fm}^{2}$. Weighting the $s$ by $-1 / 3$ and $\bar{s}$ by $+1 / 3$, we find that the mean square charge radius of strange quarks is between $(-0.36+1.0) / 3$ and $(-0.5+1.4) / 3$, that is in the range $(0.21,0.30) \mathrm{fm}^{2}$, times the probability for finding the $K \Lambda$ configuration.

To calculate $G_{E}^{s}$ at $Q^{2}=0.1 \mathrm{GeV}^{2}=2.5 \mathrm{fm}^{-2}$, we assume that the term $-Q^{2}<r^{2}>/ 6$ dominates and finally multiply by -3 to agree with the usual convention of removing the strange quark charge. This yields $G_{E}^{s} \in$ $(+0.01,+0.02)$. It is definitely small and definitely positive for the very clear physical reasons that the $K \Lambda$ probability is small and that the kaon cloud extends outside the $\Lambda$. A comparison with the currently preferred fit to the existing world data ${ }^{20}$ reveals that this estimate is in agreement at the $1 \sigma$ level, although it is nominally of opposite sign. 


\subsection{Strangeness magnetic moment}

Because orbital angular momentum is quantized, the contribution to the magnetic moment from the $\bar{s}$ in the kaon cloud is much less model dependent. The Clebsch-Gordon coefficients show that in a spin-up proton the probability of a spin down (up) $\Lambda$, accompanied by a kaon with orbital angular momentum $+1(0)$, is $2 / 3(1 / 3)$. We also know the magnetic moment of the $\Lambda$ and that it is dominated by the magnetic moment of the $s$ quark. Hence the total strangeness magnetic moment of the proton is $-3 \times P_{K \Lambda} \times 2 / 3 \times(+0.6+1 / 3)-3 \times P_{K \Lambda} \times 1 / 3 \times(-0.6+0)$, where the terms in brackets are, respectively, the magnetic moment of the spin down (up) $\Lambda$ and the magnetic moment of the charge $+1 / 3 \bar{s}$ quark with one unit (or zero units) of orbital angular momentum. The nett result, namely $G_{M}^{s}=-0.063 \mu_{N}$, is reasonably close to the best lattice QCD estimate noted above, that is $G_{M}^{s}=-0.046 \pm 0.019 \mu_{N}$. From the point of view of this "back of the envelope" estimate, the lattice result clearly has both a natural magnitude and sign. It is very hard to see how the result could change much in either magnitude or sign unless the physical picture presented here is totally incorrect. Given the remarks concerning our present understanding of hadron structure based on experience with the study of chiral extrapolation and lattice QCD data, this seems unlikely.

\section{Impact of Charge Symmetry Violation on $G_{M}^{s}$}

In the spirit of Refs. ${ }^{25,26}$, we use $p, n, u^{p}$ etc., to denote the magnetic moment of that baryon or, in the case of a quark, to denote the valence quark sector contribution of that flavor to that baryon if that quark had unit charge. A valence quark sector contribution is depicted in the lefthand diagram of Fig. 1. We also denote the total contribution of $u, d$ and $s$ quarks in a "disconnected loop" in baryon $B$, depicted in the right-hand diagram of Fig. 1, as $O_{B}$. By determining $O_{p}$, the strangeness magnetic moment of the proton can be obtained by calculating the ratio of strange to non-strange loop contributions.

The magnetic moments of the octet baryons satisfy:

$$
\begin{array}{rlrl}
p & =e_{u} u^{p}+e_{d} d^{p}+O_{p} ; & n & =e_{d} d^{n}+e_{u} u^{n}+O_{n}, \\
\Sigma^{+} & =e_{u} u^{\Sigma^{+}}+e_{s} s^{\Sigma^{+}}+O_{\Sigma^{+}} ; \Sigma^{-}=e_{d} d^{\Sigma^{-}}+e_{s} s^{\Sigma^{-}}+O_{\Sigma^{-}}, \\
\Xi^{0} & =e_{s} s^{\Xi^{0}}+e_{u} u^{\Xi^{0}}+O_{\Xi^{0}} ; \quad \Xi^{-}=e_{s} s^{\Xi^{-}}+e_{d} u^{\Xi^{-}}+O_{\Xi^{-}} .
\end{array}
$$

Having removed the charge factors from the valence quark contributions to 

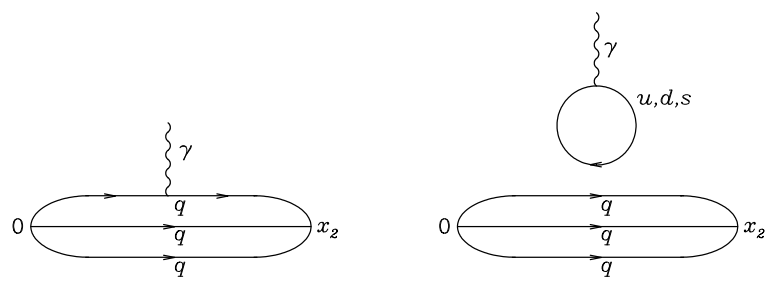

Figure 1. Diagrams illustrating the two topologically different insertions of the current within the framework of lattice QCD. In full QCD these diagrams are dressed with an arbitrary number of gluons and additional quark loops.

baryon magnetic moments, it is usually at this point that charge symmetry is invoked to provide equivalence between the doubly-represented $u$-quark sector of the proton $u^{p}$ and the doubly-represented $d$-quark sector of the neutron, $d^{n}$. Similarly, $u^{\Sigma^{+}}$is taken to be equal to $d^{\Sigma^{-}}$, and $u^{\Xi^{0}}$ is taken to be equal to $d^{\Xi^{-}}$. However, current quark mass differences of a few $\mathrm{MeV}$ and electromagnetic effects will act to violate these equalities. In these cases, charge symmetry violation (CSV) in the quark flavor being probed by the electromagnetic current is directly related to the differences observed in baryon properties.

However, indirect environmental effects are also induced through CSV. For example, even though it is the same strange quark that appears in $\Sigma^{+}$ and $\Sigma^{-}$, its contributions to the baryon moment will differ due to subtle differences in the environment of the strange quark. Similar environmental effects will provide subtle violations of $s^{\Xi^{0}}=s^{\Xi^{-}}, O_{p}=O_{n}, O_{\Sigma^{+}}=O_{\Sigma^{-}}$ and $O_{\Xi^{0}}=O_{\Xi^{-}}$.

Introducing $\Delta_{B}$ to denote the contribution to the magnetic moment of baryon $B$ having its origin in CSV, Eqs. (1) take the exact forms

$$
\begin{array}{rlrl}
p & =e_{u} u^{p}+e_{d} d^{p}+O_{p} ; & n & =e_{d} u^{p}+e_{u} d^{p}+O_{p}-\Delta_{n}, \\
\Sigma^{+} & =e_{u} u^{\Sigma^{+}}+e_{s} s^{\Sigma^{+}}+O_{\Sigma^{+}} ; \Sigma^{-} & =e_{d} u^{\Sigma^{+}}+e_{s} s^{\Sigma^{+}}+O_{\Sigma^{+}}-\Delta_{\Sigma^{-}}, \\
\Xi^{0} & =e_{s} s^{\Xi^{0}}+e_{u} u^{\Xi^{0}}+O_{\Xi^{0}} ; & \Xi^{-} & =e_{s} s^{\Xi^{0}}+e_{d} u^{\Xi^{0}}+O_{\Xi^{0}}-\Delta_{\Xi^{-}} .
\end{array}
$$

While we have elected to write the right-hand expressions of Eqs. (2) in terms of quantities in the left-hand expressions and $\Delta_{B}$, we note that one could have done the opposite and this will be important in quantifying $\Delta_{B}$.

The total sea-quark loop contribution to the proton magnetic moment, $O_{p}$, includes sea-quark-loop contributions from $u, d$ and $s$ quarks (right- 
hand side of Fig. 1). By definition

$$
\begin{aligned}
O_{p} & =\frac{2}{3} \ell^{\ell} G_{M}^{u}-\frac{1}{3} \ell^{\ell} G_{M}^{d}-\frac{1}{3}{ }^{\ell} G_{M}^{s}, \\
& =\frac{1}{3}{ }^{\ell} G_{M}^{d}-\frac{1}{3} \ell^{\ell} G_{M}^{s}-\frac{2}{3} \Delta_{\text {loop }},
\end{aligned}
$$

where we have introduced

$$
{ }^{\ell} G_{M}^{u}={ }^{\ell} G_{M}^{d}-\Delta_{\text {loop }},
$$

with $\Delta_{\text {loop }}$ accounting for differences in the $u$ and $d$ sea-quark loop contributions to the proton due to direct CSV.

Introducing, in the usual fashion, the ratio of $s$ - to $d$-quark loop contributions, ${ }^{\ell} R_{d}^{s} \equiv{ }^{\ell} G_{M}^{s} /{ }^{\ell} G_{M}^{d}$, Eq. (4) provides

$$
O_{p}=\frac{{ }^{\ell} G_{M}^{s}}{3}\left(\frac{1-{ }^{\ell} R_{d}^{s}}{{ }^{\ell} R_{d}^{s}}\right)-\frac{2}{3} \Delta_{\text {loop }},
$$

The established approach ${ }^{16}$ centres around two equations for the strangeness magnetic moment of the nucleon, $G_{M}^{s}$, obtained from linear combinations of the above. Including the $\Delta_{B}$ terms to account for CSV, one has the exact relations

$$
\begin{aligned}
& G_{M}^{s}=\left(\frac{{ }^{\ell} R_{d}^{s}}{1-{ }^{\ell} R_{d}^{s}}\right)\left[2 p+2 \Delta_{\text {loop }}+n+\Delta_{n}-\frac{u^{p}}{u^{\Sigma}}\left(\Sigma^{+}-\Sigma^{-}-\Delta_{\Sigma^{-}}\right)\right], \\
& G_{M}^{s}=\left(\frac{{ }^{\ell} R_{d}^{s}}{1-{ }^{\ell} R_{d}^{s}}\right)\left[p+2 \Delta_{\text {loop }}+2 n+2 \Delta_{n}-\frac{u^{n}}{u^{\Xi}}\left(\Xi^{0}-\Xi^{-}-\Delta_{\Xi^{-}}\right)\right] .
\end{aligned}
$$

The ratios $u^{p} / u^{\Sigma}$ and $u^{n} / u^{\Xi}$ are ratios of valence-quark contributions to baryon magnetic moments in full QCD as depicted in the left-hand diagram of Fig. 1. The latter are determined by lattice QCD calculations and finite range regularization effective field theory techniques ${ }^{16}$ with the results

$$
\frac{u^{p}}{u^{\Sigma}}=1.092 \pm 0.030 \text { and } \frac{u^{n}}{u^{\Xi}}=1.254 \pm 0.124 \text {. }
$$

The ratio of $s$ - and $d$-quark sea-quark loop contributions, ${ }^{\ell} R_{d}^{s} \equiv G_{M}^{s} /{ }^{\ell} G_{M}^{d}$, has been estimated conservatively ${ }^{16,28}$ as $0.139 \pm 0.042$.

Tests of CSV suggest that it is typically smaller than a $1 \%$ effect in baryon properties. The structure of Eqs. (7) and (8) suggests that a good estimate of the systematic uncertainty in $G_{M}^{s}$ would be provided by taking the CSV terms $\Delta_{B}$ to represent uncertainties with a magnitude of $1 \%$ of the associated baryon moment.

As discussed following Eqs. (2), the CSV corrections $\Delta_{\Sigma^{-}}$, and $\Delta_{\Xi^{-}}$ could equally well have been represented on the left-hand expressions of 
Eqs. (2) as $\Delta_{\Sigma^{+}}$, and $\Delta_{\Xi^{0}}$. Hence we will replace $\Delta_{\Sigma^{-}}$, and $\Delta_{\Xi^{-}}$in Eqs. (7) and (8) with $\Delta_{\Sigma}$, and $\Delta_{\Xi}$ representing the average $1 \%$ uncertainties of the hyperon charge states. Our focus on $O_{p}$ for strangeness in the proton, does not allow a similar symmetry for the nucleon.

Since the underlying mechanisms giving rise to CSV, as represented by $\Delta_{B}$, are different for each baryon, the uncertainties are accumulated in quadrature. Focusing on Eq. (8) where the error is largest, this provides a CSV uncertainty of $0.011 \mu_{N}$ to $G_{M}^{s}$. Given the already large error on $G_{M}^{s}=-0.046 \pm 0.019 \mu_{N}$ associated with statistical, scale determination, chiral correction and ${ }^{\ell} R_{d}^{s}$ uncertainties ${ }^{28}$, this additional CSV uncertainty has only a small effect on the final error estimate. Adding the CSV uncertainty in quadrature provides a total uncertainty of $0.022 \mu_{N}$.

\section{Concluding Remarks}

In the light of recent insights into hadron structure based on lattice QCD and the associated work on chiral extrapolation using a finite range regulator, we have explained how to quickly and easily estimate the strangeness electric and magnetic form factors of the proton. The resulting ranges,

$G_{E}^{s}\left(0.1 \mathrm{GeV}^{2}\right) \in(+0.01,+0.02)$ and $G_{M}^{s}=-0.063 \mu_{N}$ are relatively small, certainly challenging for our experimental colleagues, but consistent within 95\% CL with current world data. The latter is also in remarkable agreement with the recent determination based on lattice QCD.

We also explored the size of systematic uncertainties associated with charge symmetry violation in the recent precise determination of the strange magnetic moment of the proton ${ }^{16}$. We find CSV acts to increase the error estimate by $0.003 \mu_{N}$ such that $G_{M}^{s}=-0.046 \pm 0.022 \mu_{N}$. Hence even accounting for CSV in the approach, one still has a two-sigma signal for the sign of the strange magnetic moment of the proton.

In conclusion, this is a crucial point in the history of the study of hadron structure. For the first time we have useful guidance from non-perturbative QCD using the methods of lattice QCD and chiral extrapolation. These rigorous calculations can be given life through the sort of simple physical model described here, which nevertheless permits semi-quantitative calculation. At the same time we have new experimental capabilities to accurately measure the role of non-valence quarks in static properties which can be used to test our new found theoretical advances. 


\section{Acknowledgements}

This work is supported by the Australian Research Council and by DOE contract DE-AC05-84ER40150, under which SURA operates Jefferson Laboratory.

\section{References}

1. W. Detmold, D. B. Leinweber, W. Melnitchouk, A. W. Thomas and S. V. Wright, Pramana 57, 251 (2001) [arXiv:nucl-th/0104043].

2. R. D. Young, D. B. Leinweber, A. W. Thomas and S. V. Wright, Phys. Rev. D 66, 094507 (2002) [arXiv:hep-lat/0205017].

3. D. B. Leinweber, D. H. Lu and A. W. Thomas, Phys. Rev. D 60, 034014 (1999) [arXiv:hep-lat/9810005].

4. V. V. Flambaum et al., Phys. Rev. D 69, 115006 (2004).

5. I. C. Cloet, D. B. Leinweber and A. W. Thomas, Phys. Rev. C 65, 062201 (2002) [arXiv:hep-ph/0203023].

6. W. Detmold, W. Melnitchouk and A. W. Thomas, Eur. Phys. J. directC 3, 13 (2001) [arXiv:hep-lat/0108002].

7. D. B. Leinweber, et al., Phys. Rev. D 61, 074502 (2000) [hep-lat/9906027].

8. A. W. Thomas, Adv. Nucl. Phys. 13, 1 (1984).

9. G. A. Miller, Int. Rev. Nucl. Phys. 1, 189 (1984).

10. D. I. Glazier et al., Eur. Phys. J. A 24, 101 (2005) [arXiv:nucl-ex/0410026].

11. J. M. Finn [E93038 Collaboration], FizikaB 13 (2004) 545.

12. D. H. Lu, A. W. Thomas and A. G. Williams, Phys. Rev. C 57, 2628 (1998)

13. K. Goeke, J. Ossmann, P. Schweitzer and A. Silva, arXiv:hep-lat/0505010.

14. A.W. Schreiber, A.I. Signal and A.W. Thomas, Phys. Rev. D 44 (1991) 2653.

15. B. Dressler, et al., arXiv:hep-ph/9809487.

16. D. B. Leinweber et al., Phys. Rev. Lett. 94, 212001 (2005)

17. D. T. Spayde et al. [SAMPLE Collaboration], Phys. Rev. Lett. 84, 1106 (2000) [arXiv:nucl-ex/9909010].

18. E. J. Beise, M. L. Pitt and D. T. Spayde, Prog. Part. Nucl. Phys. 54, 289 (2005) [arXiv:nucl-ex/0412054].

19. F. E. Maas et al., Phys. Rev. Lett. 94, 082001 (2005).

20. D. S. Armstrong et al. [G0 Collaboration], Phys. Rev. Lett. 95, 092001(2005).

21. K. A. Aniol et al. [HAPPEX Collaboration], arXiv:nucl-ex/0506011.

22. J. Speth and A. W. Thomas, Adv. Nucl. Phys. 24, 83 (1997).

23. A. W. Thomas, S. Theberge and G. A. Miller, Phys. Rev. D 24, 216 (1981).

24. A. W. Thomas, J. D. Ashley, D. B. Leinweber and R. D. Young, J. Phys. Conf. Ser. 9, 321 (2005).

25. D. B. Leinweber, Phys. Rev. D 53, 5115 (1996) [arXiv:hep-ph/9512319].

26. D. B. Leinweber and A. W. Thomas, Phys. Rev. D 62, 074505 (2000).

27. I. Eschrich et al. [SELEX Collaboration], Phys. Lett. B 522, 233 (2001).

28. D. B. Leinweber, et al., Eur. Phys. J. A 24S2, 79 (2005). 